\title{
A NEW SYNTHETIC MYDRIATIC*
}

\author{
BY \\ IDA MANN \\ OXFORD
}

OwING to the serious shortage of atropine in England during $1942-43$, work on the synthesis of mydriatics was undertaken by Drs. Ing and Ford-Moore at the Dyson Perrins Laboratory, Oxford. A number of substances were produced and tested experimentally and clinically. One of these, which came to be generally known by its code name, E.3, proved of great clinical value and is likely to be of permanent use to ophthalmologists.

This substance is the benzilic ester of hydroxy ethyl-dimethylethyl-ammonium chloride, its formula being :

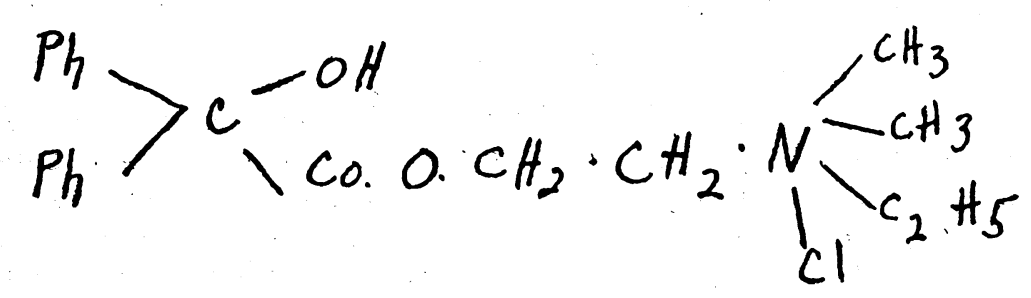

A 1 per cent. solution was used for trial. A drop of this in the eye produced mydriasis and cycloplegia in monkeys. No general symptoms were apparent. Toxicity trials in mice were satisfactory, and in cats the substance produced sleepiness, whereas atropine leads to excitation and convulsions. Clinical trials on man were therefore begun.

The patients can be divided into 4 groups. In the first group, E.3 was used on normal, healthy eyes to test its mydriatic and cycloplegic action. In the second group, it was used for treatment of diseases for which atropine would normally be given. In the third group, it was used as a substitute for atropine in cases in which atropine or hyoscine had produced general symptoms, and in the fourth group it was used in cases known to be sensitive to atropine or hyoscine and to show allergic eczema of the lids (atropine irritation) from their use.

Fifty-eight patients were studied.

Group 1. Healthy eyes.-Two drops of a 1 per cent. solution of E.3 were dropped into one or both eyes of 4 normal individuals

* Received for publication, October 22, 1945. 
at an interval of a quarter of an hour. Their ages were respectively, $2,8,59$ and 76 years. In the youngest child dilatation of the pupil was slow and was not quite complete in an hour. This was very similar to the expected action of atropine, which is slow in young children. In the boy aged 8 years, full mydriasis and paralysis of accommodation were reached in less than an hour. In the two older patients, dilatation began in twenty minutes and was complete in thirty minutes from the time of the first drop. Eserine 0.5 per cent. was then dropped into the eye twice at ten minute intervals. In the patient aged 59 years, the pupil had regained its previous size (but was not pinpoint) in twenty minutes. In the older patient, the eserine had no effect. The pupil remained dilated until the following morning. The tension was normal and two applications of eserine then restored the pupil to normal (though it did not become pinpoint).

It would appear therefore that E.3 can be used for diagnostic and refractive purposes in the same way as atropine. Its action is more easily reversed by eserine than is that of atropine, but in old people it is not so safe as is homatropine. It appears to lie between atropine and homatropine in its strength of action on normal eyes. None of the patients made any complaint of discomfort from its use.

Group 2. Treatment cases.-E.3 was next used on 6 cases of disease. Three of them were corneal ulcers. In one case atropine, and in another oily homatropine and cocaine had been used once at the beginning of the treatment; in the third, E.3 was used throughout. All three ulcers healed, two after a week's use of E.3, the third after 14 days. One of the remaining three patients was a case of tuberculous cyclitis who had been on atropine for some time. This was changed to E.3. The effect appeared to be similar, though more frequent instillations were necessary to maintain maximum dilatation, the best result being obtained if E.3 was used three times a day. Two operation cases were then treated, one of capsulotomy, in which E.3 was used before and twice a day for four days after operation with a good result, and the other, a cataract extraction in which atropine was used from the beginning. This eye was slow in settling down, and E.3 was given twice a day for two months with a final good result and no ill effects from its continued use.

Group 3. Cases with general toxic symptoms from atropine and hyoscine.-This group contained 6 patients (two chronic cyclitis, two post-operative cyclitis, and two cataract extractions, one in a diabetic). All these patients had previously been treated with atropine or hyoscine, which had produced very unpleasant general symptoms, dryness of the mouth, itching of the skin, general discomfort and, in 3 cases, mental confusion. None of 
them had shown any local signs of atropine irritation. The two cases of cyclitis were treated with E.3 twice or three times daily, one for six months, the other for two months with good local results and no recurrence of general symptoms. The two cases of post-operative cyclitis used E.3 drops twice a day for about six weeks with no general symptoms. The two cases of cataract extraction were patients who had had severe general symptoms from atropine and hyoscine used after a previous extraction. In both cases E.3 was used for the second eye, in one case for eight weeks and in the other for four without any ill effects, the mydriasis being satisfactory, and the patients making no complaint of local or general symptoms.

E.3 is therefore without systemic toxic effects in cases showing general idiosyncrasy to atropine and hyoscine.

Group 4. Patients known to have atropine irritation.-This group contains 42 cases and is by far the most interesting, as it shows that we now possess in E.3 a mydriatic of so completely different a chemical composition from atropine that it can safely be used in cases of severe atropine irritation.

In the 42 cases of atropine irritation under review, 16 had shown severe allergic eczema of the lids and face in less than a week after beginning treatment with atropine. Some of these were sensitive to homatropine and to hyoscine as well. In others, these were not tried, E.3 being substituted as soon as the atropine irritation appeared. In 17 cases the irritation did not appear until the patient had used atropine for more than a week. In the remaining 9 cases, the exact length of time of treatment with atropine before the irritation began is not known.

In all these cases, E.3 was substituted for atropine without waiting for the irritation to subside. In them all the patients experienced relief at once; there was steady subsidence of the oedema and itching and however long (in one case up to seven months) the E.3 was continued, there was no return of the symptoms.

Although a much larger number of cases will be required before we are certain that E.3 never produces an allergic reaction, we can certainly say from these 42 patients that it already gives us a very efficient non-irritating substitute for atropine and with a stronger mydriatic action than homatropine or hyoscine. In most cases it was necessary to instil it twice a day and in some three times, but no symptoms were complained of and the course of the disease being treated was what one would have expected if atropine had been used.

The diseases from which these 42 patients were suffering are shown in the following table, as well as the time they were using E.3:- 


\section{Cases with atropine irritations}

Type of case :

Corneal ulcer

Interstitial keratitis

Delayed mustard gas keratitis

Scleritis

Endophthalmitis

Atropine irritation developed in :

Less than 1 week ... 16 cases

More than 1 week ... 17 cases

$\begin{array}{llll}\text { Uncertain } & \ldots & \ldots & 9\end{array}$ cases

Total $\quad \ldots \overline{42}$

Sympathetic ophthalmitis

Acute iritis

Chronic irido-cyclitis

Cataract extraction

Capsulotomy

Post-cataract irido-cyclitis

Treated with E.3 without irritation for:

Less than 1 week ... 6 cases One week to 3 months 32 cases More than 3 months ... 4 cases

Total

\section{Summary}

The synthetic mydriatic known as E.3 is non-irritating, nontoxic and in action on the eye lies between atropine and homatropine. It is in all respects suitable as a substitute for atropine though it may have to be used more frequently. .

\section{OCULAR FINDINGS IN TROPICAL TYPHUS* (Tsutsugamushi or Japanese River Fever)}

$\mathrm{BY}$

E. A. Donegan, Captain, R.A.M.C.

Tropical typhus or Tsutsugamushi, Japanese river fever or scrub typhus has long been recognised as very closely related to the more widely known louse-borne typhus met with in the Balkans and Russia during the War 1914-1918 and again in this War in Italy, and also to the tick borne typhus of world wide distribution.

It was first described in 1878. In Mandalay, 1925, cases occurred in a military camp (Fletcher) and were divided into two types : a.W. form or urban type and a.K. form found in country districts and plantations. It is probable that the $\mathrm{W}$. form is identical with Brill's disease, which is flea borne. A complete study of tropical typhus was carried out by Anigstein, 1933.

The causative organism is Rickettsia orientalis, its insect vector

* Received for publication, October 4, 1945. 